\title{
A Graph and its Com- plement with Specified Properties. IV. Counting Self-Complementary Blocks
}

Jin Akiyama

NIPPON IKA UNIVERSITY

KAWASAKI, JAPAN

Frank Harary

THE UNIVERSITY OF MICHIGAN

ANN ARBOR, MICHIGAN

Dedicated to Robert W. Robinson

\section{ABSTRACT}

In this series, we investigate the conditions under which both a graph $G$ and its complement $\bar{G}$ possess certain specified properties. We now characterize all the graphs $G$ such that both $G$ and $\bar{G}$ have the same number of endpoints, and find that this number can only be 0 or 1 or 2 . As a consequence, we are able to enumerate the self-complementary blocks.

\section{NOTATIONS AND BACKGROUND}

In the first paper [1] in this series, we found all graphs $G$ such that both $G$ and its complement $\bar{G}$ have connectivity 1 , and other properties. In the second paper [2], we determined the graphs $G$ for which $G$ and $\bar{G}$ are obtained from some graph by the same unary operation. More recently [3] we characterized the graphs such that both $G$ and $\bar{G}$ have the same girth and the same circumference 3 or 4 .

An endpoint of graph has degree 1 . We denote the number of endpoints in $G$ by $e=e(G)$ and in $\bar{G}$ by $\bar{e}$. We characterize all the graphs $G$ with $e=\bar{e}(\geq 2)$ in the next section, and count the number of selfcomplementary blocks in the last section.

Journal of Graph Theory. Vol. 5 (1981) 103-107

(1) 1981 by John Wiley \& Sons, Inc. CCC 0364-9024/81/010103-05\$01.00 
Following the notation and terminology of [5], we define the join $G_{1}+G_{2}$ of two graphs to be the union of $G_{1}$ and $G_{2}$ with the complete bigraph having point sets $V_{1}$ and $V_{2}$, and the corona $G \circ H$ of two graphs $G$ with $p$ points $v_{i}$ and $H$ is obtained from $G$ and $p$ copies of $H$ by joining each point $v_{i}$ of $G$ with all the points of the $i$ th copy of $H$. For our result later we need a ternary operation written $F+G \circ H$ which is defined in [3] as the union of the join $F+G$ with the corona $G \circ H$. Thus this resembles the composition of the path $P_{3}$ not with just one other graph but with three graphs, one for each point, for example, Figure 1 illustrates the graph $A=K_{1}+K_{2} \circ K_{1}$.

\section{ENDPOINTS}

Let $g_{p}$ be the number of graphs of order $p$.

Lemma 1. For $n \geq 1$, the mapping $F \rightarrow F+K_{n} \circ K_{1}$ which takes graphs $F$ of order $p$ to graphs $G=F+K_{n} \circ K_{1}$ of order $p+2 n$ is one-to-one.

Proof. Suppose $G$ can be written in the form $F+K_{n} \circ K_{1}$. We will show that $F$ is uniquely recoverable from $G$. Let $S$ be the set of points of $G$ which are adjacent to endpoints. Clearly $S$ is the point set of the distinguished subgraph $K_{n}$. Let $H$ be the subgraph induced by $V(G)-S$. Then $H$ has at least $n$ isolates, and removing exactly $n$ isolates from $H$ leaves $F$. 1

Lemma 2. If $G$ has two endpoints, then $\bar{G}$ has at most two endpoints.

Proof. Let $v_{0}$ and $v_{1}$ be two endpoints of $G$, adjacent to $u_{0}$ and $u_{1}$, respectively. Then obviously the only candidates for endpoints in $\bar{G}$ are $u_{0}$ and $u_{1}$.

Theorem 1. A graph $G$ of order $p \geq 4$ has $e=\bar{e}=2$ iff $G$ is of the form $F+K_{2} \circ K_{1}$, where $F$ is a graph of order $p-4$.

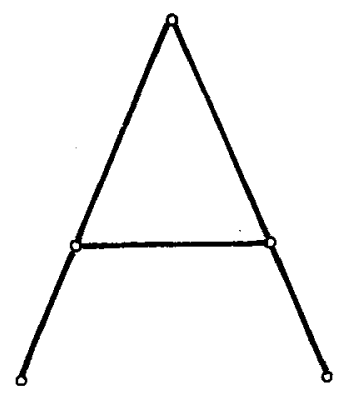

FIGURE 1. $A=K_{1}+K_{2} \circ K_{1}$ 
Proof. If $e=\bar{e}=2$, then $G$ has exactly two points $v_{0}$ and $v_{1}$ of degree $p-2$ and exactly two points $u_{0}$ and $u_{1}$ of degree 1 , where $u_{0}, u_{1}$ are not adjacent to $v_{0}, v_{1}$, respectively. Since $\operatorname{deg} v_{0}=\operatorname{deg} v_{1}=p-2, v_{i}$ is adjacent to every point other than $u_{i}$ for $i=0,1$. On the other hand, $u_{i}$ is not adjacent to any point other than $v_{1-i}$ for $i=0,1$, since $\operatorname{deg} u_{0}=\operatorname{deg} u_{1}=$ 1 . Denote by $F$ the subgraph of $G$ induced by the point set $V(G)-$ $\left\{v_{0}, v_{1}, u_{0}, u_{1}\right\}$. Then in $G$ any point $v$ of $F$ must be adjacent to both $v_{0}$ and $v_{1}$ which are adjacent to each other by the above observations. Thus $G$ is a graph of the form $F+K_{2} \circ K_{1}$.

The converse follows immediately from the proof of Lemma 1. I

Corollary 1. The number of graphs of order $p$ with $e=\bar{e}=2$ is $g_{p-4}$.

Proof. By Theorem $1, G$ is of the form $F+K_{2} \circ K_{1}$ where $F$ has $p-4$ points. Hence by the $1-1$ correspondence of Lemma 1 , the number of graphs $G$ with $e=\bar{e}=2$ is $g_{p-4}$.

Corollary 2. All graphs with $e=\bar{e}=2$ have diameter 3 .

Proof. The maximum distance between two points of $F+K_{2} \circ K_{1}$ is 3, as this is the distance between the two endpoints.

\section{SELF-COMPLEMENTARY GRAPHS}

A graph $G$ is self-complementary (or briefly, s-c) if it is isomorphic to its complement $\bar{G}$. The isomorphism between $G$ and $\bar{G}$ can be represented as a permutation, $\alpha$, on $V(G)$. We will write $\alpha(G)=\bar{G}$ and call $\alpha$ a complementing permutation for $G$ as in Gibbs [6]. We will assume that all permutations are expressed as the product of disjoint cycles. We first state the result obtained independently by Ringel [8] and Sachs [10], which gives the cycle structure of a complementing permutation.

Theorem RS. If $G$ is s-c of order $p$ and $\alpha(G)=\bar{G}$, then if $p \equiv 0(\bmod 4)$, each cycle of $\alpha$ has length divisible by 4 and if $p \equiv 1(\bmod 4), \alpha$ has exactly one cycle of length 1 and all other cycles have length divisible by 4.

We begin with the result concerning the number of endpoints of a s-c graph, which was communicated to us by R. W. Robinson and proved nicely by one of the referees.

Lemma 3. A self-complementary graph does not have exactly one endpoint. 
Proof. Suppose $G$ is s-c with a unique point of degree 1 . Then $G$ must have a unique point of degree $p-2$ and these observations hold for $\bar{G}$ as well. In $G$ let $\operatorname{deg} v_{1}=1$ and $\operatorname{deg} v_{2}=p-2$. Hence in $\bar{G}$, $\operatorname{deg} v_{1}=$ $p-2$ and $\operatorname{deg} v_{2}=1$. But $v_{1}$ and $v_{2}$ are adjacent in exactly one of $G$ and $\bar{G}$, a contradiction.

We now characterize all s-c graphs with two endpoints.

Lemma 4. All s-c graphs of order $p+4$ having two endpoints can be constructed using the ternary operation $G=F+K_{2} \circ K_{1}$, where $F$ is a s-c graph of order $p$.

Proof. Let $G$ be any s-c graph of order $p+4$ having 2 endpoints. Since $G \cong \bar{G}$ and $G$ has exactly 2 endpoints, we know that $G$ is of the form $F+K_{2} \circ K_{1}$ for some graph $F$ of order $p$ by Theorem 1. On the other hand, it is easy to see that $G=F+K_{2} \circ K_{1}$ is s-c iff $F$ is s-c. Thus, $G$ can be constructed using the ternary operation $G=F+K_{2} \circ K_{1}$ for some s-c graph $F$ of order $p$. I

We denote by $s_{\mathrm{p}}$ the number of all s-c graphs of order $p$ and by $s_{p}^{\prime \prime}$ the number of s-c graphs of order $p$ which have 2 endpoints. Since the ternary operation $G=F+K_{2} \circ K_{1}$ is $1-1$ as proved in Lemma 1 , we have the following equality from Lemma 4 .

Lemma 5. For any positive integer $p$,

$$
s_{p+4}^{\prime \prime}=s_{p} \text {. }
$$

Recall [5, p. 24] that $G$ is a block if $G$ is connected and has no cutpoint. The number of blocks was determined by Robinson [9]. Our object is to derive the number of self-complementary blocks.

Lemma 6. If $G$ is a s-c graph with no endpoints, then $G$ is a block.

Proof. Assume that $G$ is s-c with no endpoints but has a cutpoint $v$. The removal of $v$ from $G$ results in a subgraph with at least 2 components. Let $G_{1}$ be a component of $G-v$ and let $G-v=G_{1} \cup G_{2}$. Thus $\overline{G-v}$ contains a complete spanning bigraph B whose point sets are $V\left(G_{1}\right)$ and $V\left(G_{2}\right)$. The cardinalities of both $V\left(G_{1}\right)$ and $V\left(G_{2}\right)$ are at least 2 by the hypothesis that $G$ has no endpoints. Therefore $\bar{G}$ is 2 -connected and hence $G=\bar{G}$ cannot have a cutpoint, a contradiction.

Read [7] found a formula for the number of self-complementary graphs $s_{\mathrm{p}}$. Frucht and Harary [4] derived an alternative equation. We now see how to count $\mathrm{s}-\mathrm{c}$ blocks in terms of the numbers $s_{\mathrm{p}}$.

Theorem 2. For any positive integer $p \geq 5$, the number of $s-c$ blocks of order $p$ is $s_{p}-s_{p-4}$. 
Proof. Let $G$ be a self-complementary block of order $p$, so that $p \geq 5$. By Lemmas 3 and 6, the number of s-c blocks equals $s_{p}$ less the number of $\mathrm{s}-\mathrm{c}$ graphs with $e=2$. But this is $s_{\mathrm{p}-4}$ by Lemma 5 .

\section{ACKNOWLEDGMENT}

We thank Geoffrey Exoo for several helpful comments.

\section{References}

[1] J. Akiyama and F. Harary, A graph and its complement with specified properties I: Connectivity. Internat. J. Math. and Math. Sci. 2 (1979) 223-228.

[2] J. Akiyama and F. Harary, A graph and its complement with specified properties II: Unary operations. Nanta Math. To appear.

[3] J. Akiyama and F. Harary, A graph and its complement with specified properties III: Girth and circumference. Internat. J. Math. and Math. Sci. 2 (1979) 685-692.

[4] R. Frucht and F. Harary, Self-complementary generalized orbits of a permutation group. Canad. Math. Bull. 17 (1974) 203-208.

[5] F. Harary, Graph Theory. Addison-Wesley, Reading, MA (1969).

[6] R. A. Gibbs, Self-complementary graphs. J. Combinatorial Theory Ser. B 16 (1974) 106-123.

[7] R. C. Read, On the number of self-complementary graphs and digraphs. J. London Math. Soc. 38 (1963) 99-104.

[8] G. Ringel, Selbstkomplementäre Graphen. Arch. Math. 14 (1963) 354-358.

[9] R. W. Robinson, Enumeration of non-separable graphs. J. Combinatorial Theory 9 (1970) 327-356.

[10] H. Sachs, Über selbstkomplementäre Graphen, Publ. Math. Debrecen 9 (1962) 270-288. 